This document is confidential and is proprietary to the American Chemical Society and its authors. Do not copy or disclose without written permission. If you have received this item in error, notify the sender and delete all copies.

\title{
Black GaAs by Metal-Assisted Chemical Etching
}

\begin{tabular}{|r|l|}
\hline Journal: & ACS Applied Materials \& Interfaces \\
\hline Manuscript ID & am-2018-10370n.R1 \\
\hline Manuscript Type: & Article \\
\hline Complete List of Authors: & $\begin{array}{l}\text { Lova, Paola; Universita degli Studi di Genova, Dipartimento di Chimica e } \\
\text { Chimica Industriale } \\
\text { Robbiano, Valentina; Università degli studi di Pisa, Dipartimento di } \\
\text { ingegneria dell'informazione } \\
\text { Cacialli, Franco; University College London, London Centre for } \\
\text { Nanotechnology and Department of Physics and Astronomy } \\
\text { Comoretto, Davide; Università degli Studi di Genova, Chimica e Chimica } \\
\text { Industriale } \\
\text { Soci, Cesare; Nanyang Technological University, Physics and Applied } \\
\text { Physics }\end{array}$ \\
\hline \hline
\end{tabular}




\title{
Black GaAs by Metal-Assisted Chemical Etching
}

Paola Lova, ${ }^{1,4}$ Valentina Robbiano, ${ }^{2}$ Franco Cacialli, ${ }^{2}$ Davide Comoretto ${ }^{3}$ and Cesare Soci ${ }^{1,4, *}$

1 Energy Research Institute at NTU (ERI@N) and Interdisciplinary Graduate School, Nanyang Technological University, 50 Nanyang Avenue, Singapore 639798

2 Department of Physics and Astronomy and London Centre for Nanotechnology, University College London, WC1E 6BT, United Kingdom

3 Dipartimento di Chimica e Chimica Industriale, Università degli Studi di Genova, via Dodecaneso 31, 16121, Genova, Italy

4 School of Physical and Mathematical Sciences, Division of Physics and Applied Physics, Nanyang technological University, 21 Nanyang Link, Singapore 637371

KEYWORDS: Metal assisted chemical etching, anisotropic etching, perfect antireflection, black GaAs, III-V semiconductors.

\begin{abstract}
Large area surface microstructuring is commonly employed to suppress light reflection and enhance light absorption in silicon photovoltaic devices, photodetectors and image sensors. To date, however, there are no simple means to control the surface roughness of III-V semiconductors by chemical processes similar to the metal assisted chemical etching of black Si. Here we demonstrate anisotropic metal assisted chemical etching of GaAs wafers exploiting the lower etching rate of the monoatomic $\mathrm{Ga}<111>$ and $<311>$ planes. By studying the dependence of this process on different crystal orientations, we propose a qualitative reaction mechanism responsible for the self-limiting anisotropic etching and show that reflectance of the roughened surface of black GaAs reduces up to 50 times compared to polished wafers, nearly doubling its absorption. This method provides a new, simple and scalable way to enhance light absorption and power conversion efficiency of GaAs solar cells and photodetectors.
\end{abstract}

\section{Introduction}

Scattering and antireflective superficial layers are widely used to increase photovoltaic efficiency of inorganic semiconductor, ${ }^{1-2}$ conjugated polymer $^{3}$ and perovskite ${ }^{4-5}$ solar cells. Such layers relies on light trapping surfaces, ${ }^{6-7}$ scattering structures ${ }^{8}$ and photonic crystals, ${ }^{9-11}$ which increase the light path within the photoactive layer, enhance light absorption, and broaden the absorption spectrum. ${ }^{12}$ The fabrication 
of these structures is often accomplished by lithographic techniques, which are hard to implement within the workflow of solar cell production. Wet processes for large area antireflection or light trapping surfaces are a low-cost, easily scalable alternative to lithography. For example, metal-assisted chemical etching (MACE) is widely used to fabricate high aspect ratio nanowire array with near-zero surface reflectivity on silicon ${ }^{13-21}$ and germanium ${ }^{22}$ surfaces. MACE relies on the dissolution of a substrate operated by a mixture of oxidizing and acidic or alkaline species. ${ }^{19,}{ }^{23-24}$ In such processes, a transition metal $^{13,16,25}$ catalyses the anisotropic and directional etching of the substrate surface. ${ }^{24,32,41}$ As a result, when the catalyst is randomly dispersed on the surface, the process generates nano- and microstructured arrays $^{14,18,26-27}$ (see Supporting Information Fig. S1 for process details). So far, only few attempts were made to extend wet etching processes to structure the surface of III-V semiconductors. MACE of GaAs was demonstrated in conjunction with thermal evaporation ${ }^{28}$, or lithographic patterning of the metal catalyst ${ }^{29-41}$ by photolithography, ${ }^{16}$ nanoimprint lithography, ${ }^{42}$ and microsphere selfassembly, ${ }^{43}$ while the use of catalyst nanoparticles was shown using Au films evaporated on the GaAs surface and subsequently treated at high temperature. ${ }^{39}$ Thermal treatment can induce arsenic vacancies $^{44}$ and lead to catalyst diffusion on the GaAs surface. So far, lithographic patterning and thermal evaporation have always been necessary to achieve the desired anisotropic etching of III-V semiconductors, substantially limiting the scalability of the process. Here we demonstrate a lithography-free MACE process for structuring GaAs surfaces with near-zero reflection ${ }^{45}$ (black GaAs). The method relies on the electroless deposition of randomly dispersed gold nanoparticles cast from solution on the GaAs surface and etching in in $\mathrm{H}_{2} \mathrm{O}_{2}$ and $\mathrm{HF}$, which are entirely performed at ambient conditions. We find that the antireflective properties of black GaAs outperform those of the well-known micro-/nanostructured silicon (black $S i$ ), yielding reflectance values as low as 0.013 .

\section{Experimental Methods}

Metal-assisted chemical etching: MACE was performed on silicon doped $n$-type (111)B, (100), (211) and (110) GaAs epi-ready wafers (purchased from Axt Inc). In a typical process, gold nanoparticles are deposed on the semiconductor surface by immersion in a water solution containing $0.1 \mathrm{mM}$ of $\mathrm{AuCl}_{3}$. Samples are then blow dried and etched in $\mathrm{HF}: \mathrm{H}_{2} \mathrm{O}_{2}$ (4:1) for 10 minutes.

Reference black Si samples were fabricated by a standard two-step process of $p$-type (100) Si wafers (purchased from Latech Scientific). First, samples are etched in a solution of $\mathrm{H}_{2} \mathrm{O}_{2}: \mathrm{HF}(9: 1)$ containing $0.02 \mathrm{M}$ of $\mathrm{AgNO}_{3}$ for one hour. Then, further etching in $\mathrm{H}_{2} \mathrm{O}_{2}$ and $\mathrm{NH}_{4} \mathrm{OH}(1: 3)$ is used to remove residual catalyst and etching by-products from the surface (see Supporting Fig. S6). 
Structural and optical characterization: Microstructure and topography images of the etched surfaces were obtained by scanning electron microscopy (SEM). Data were collected by a field emission SEM Jeol JSM-6700F using acceleration voltage of $5.0 \mathrm{kV}$ and using high (SEI) and low (LEI) secondary electrons detectors. Individual SEM images were analysed to extract surface features sizes and orientations. Representations of the GaAs crystal lattice were elaborated with the software VESTA ${ }^{46}$ using data retrieved from the Crystallography Open Database. ${ }^{47}$

Reflectance was measured with a spectrophotometer (Andor Shamrock 163i) equipped with an integrating sphere (Bentham IS4-ODM-X) in the spectral range between 550 and $1100 \mathrm{~nm}$. In the 1200-2600 $\mathrm{nm}$ spectral range, data were acquired in a fibre-based optical setup with a Micropak DH2000BAL light source and an Arcoptics FT-interferometer (1000-2600 nm, resolution $\left.8 \mathrm{~cm}^{-1}\right)$.

Photoluminescence external quantum efficiency was measured as previously reported by the method reported by De Mello et al., ${ }^{48-50}$ using an integrating sphere (Avantes AvaSphere-50) coupled to a CCD spectrometer (Avantes 2048) in the 200-1100 nm spectral range. The excitation source was a red diode laser (CNI model MRL-655) with $400 \mathrm{~mW}$ power. Wavelength dependent photoluminescence measurements were performed with a spectrofluorometer (Horiba Fluorolog) equipped with a Si CCD detector. The excitation source was a Xe discharge lamp.

\section{Results and Discussion}

We developed a two-step etching process of GaAs wafers via the electroless deposition of metal nanoparticles (etching catalyst) followed by etching in an acidic oxidizing bath. Randomly dispersed Au nanoparticles are precipitated from an $\mathrm{AuCl}_{3}$ water solution on the GaAs surface by redox reaction between the $\mathrm{Au}^{3+}$ ions, which are reduced to $\mathrm{Au}^{0}$, and $\mathrm{GaAs}$, which is oxidized (see Supporting Information Fig. S2). The substrate is then immersed in a bath containing $\mathrm{H}_{2} \mathrm{O}_{2}$ and $\mathrm{HF}$ for 10 minutes to initiate the catalytic process. Figs. $1 \mathrm{a}$ and $1 \mathrm{~b}$ show the scanning electron micrographs of etched GaAs surfaces with crystallographic orientation (111)B. The topographic images (left side) show the morphology of surface features, while the compositional images (right side) highlight the presence of residual gold nanoparticles, which are also visible as brighter spots in topographic images.

Etching of the (111)B surface produces randomly oriented, faceted hillocks with heights of about 5.5 $\mu \mathrm{m}$ and width of about $5 \mu \mathrm{m}$. It can be immediately noticed that the microfacet orientation remains constant throughout the sample surface, indicating a crystal plane dependent etching rate. This is further confirmed by the SEM micrographs of etched wafers with crystal orientations (100), (211) and (110) (see Supporting Information Fig. S3 and Fig. 1d). 
Slow etch rate planes can be identified with the microfacet orientations comparing them with a representation of the GaAs crystal structure (Figs. 1c and 1f). Fig. 1c shows that the side facets of the hillocks protruding from the (111)B surface form angles of $70^{\circ}$ (blue dotted lines) or $79^{\circ}$ (green dashed lines) with the latter, corresponding to the $(1 \overline{3} 1)$ and $(1 \overline{1} 1)$ crystallographic orientations. Similarly, the angle between the facets (blue dotted lines in Fig. 1f) and the (100) plane (orange lines in Fig. 1f) have an angle of $26^{\circ}$ with respect to the (100) plane, corresponding to slow etch rate planes $(3 \overline{1} \overline{1})$ and $(\overline{1} \overline{1} \overline{1})$.
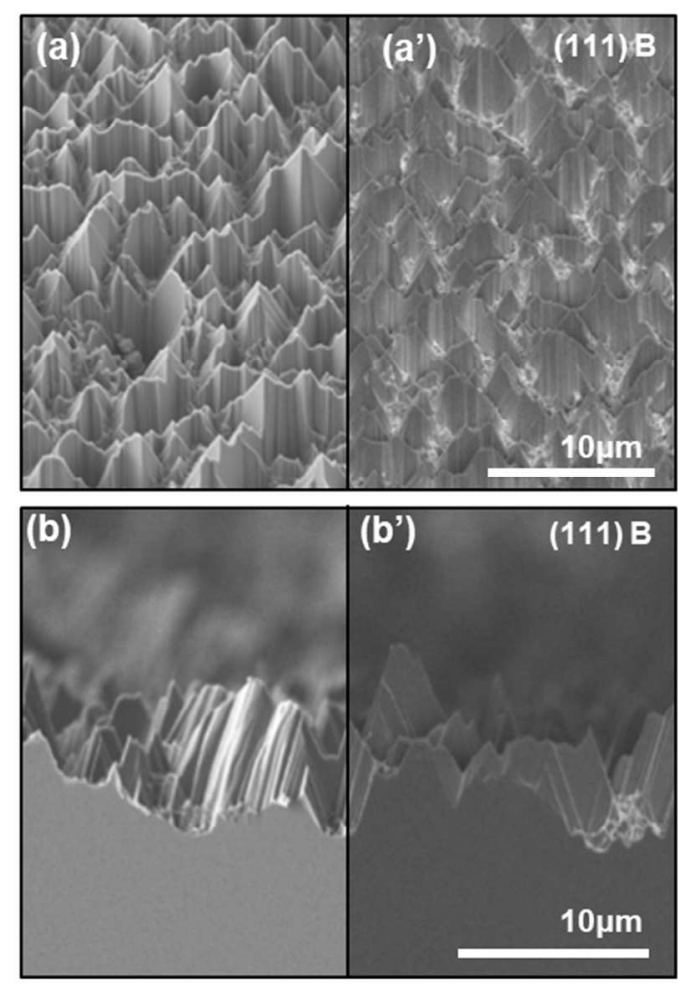

Gallium

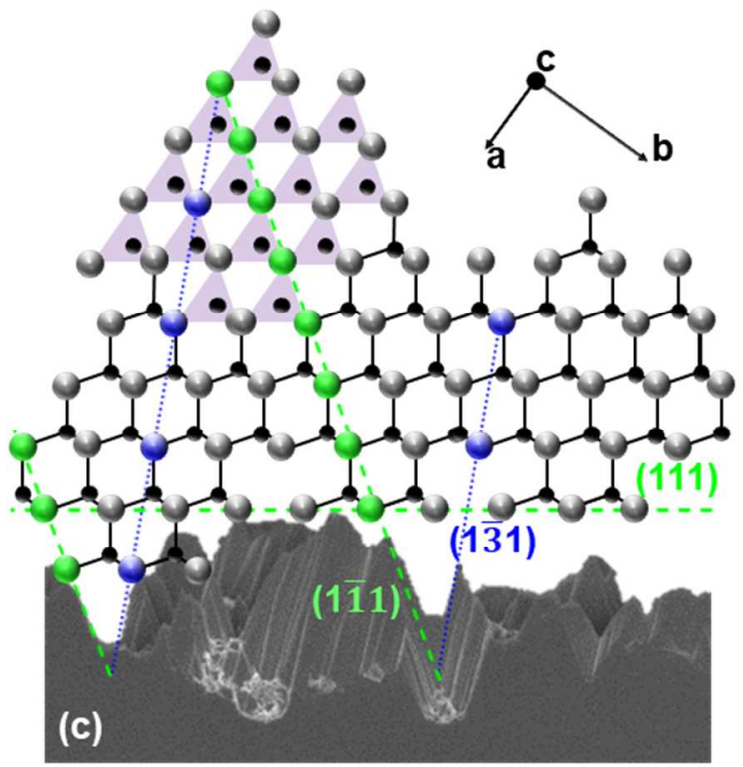

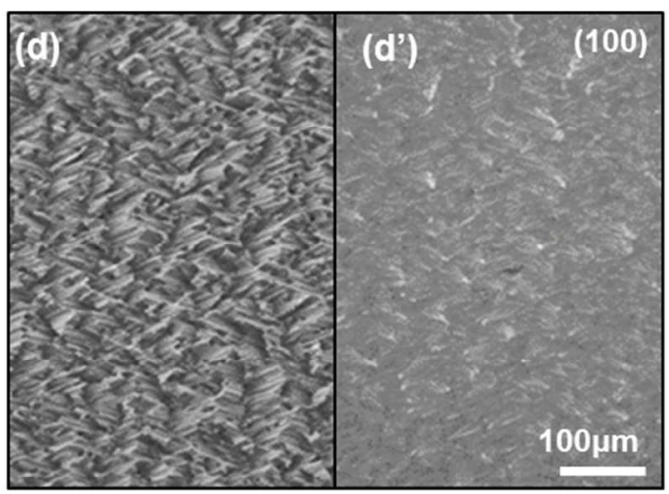

(e)

$\left(e^{\prime}\right)$

(100)

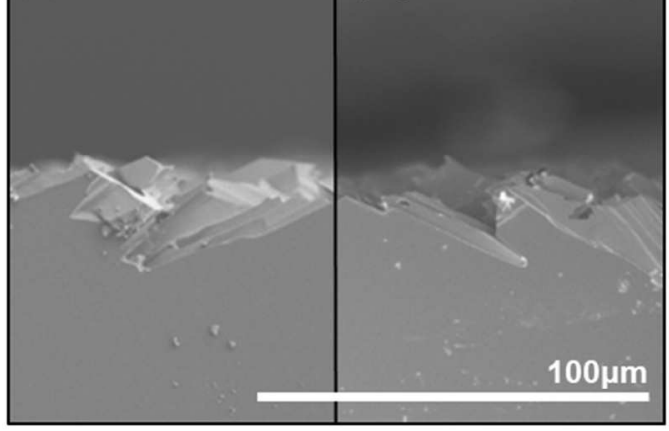
$<100>$

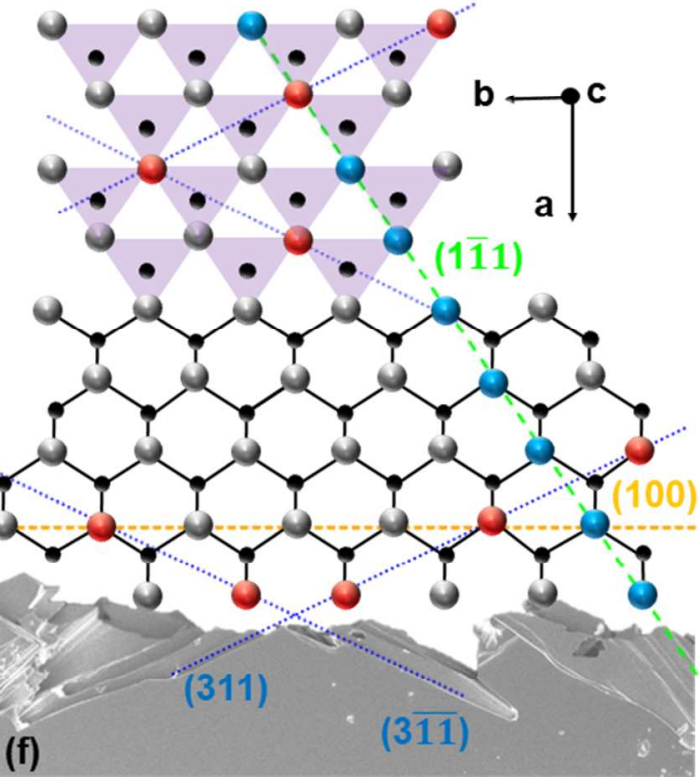


Figure 1: SEM imaging and analysis of the etched (111)B (panels a-c) and (100) (panels d-f) surfaces of GaAs. Top: tilted (a,a' and d,d') and cross-sectional views (b,b' and e,e') in topographical (a-d) and compositional (a'-d') modes. Bottom: GaAs crystal representations overlaid to the etched surface profiles (c and f).

The fact that monoatomic $<111>$ and $<311>$ planes are etched at slower rate than others can be understood considering the reactivity of individual $\mathrm{Ga}$ and As atoms. The $\mathrm{sp}_{3}$ hybridization of Ga and As atoms in the zinc-blende crystallographic structure of GaAs stabilizes gallium, which is normally trivalent, and destabilizes arsenic, which has preferential valence five in electrophilic environment. Since the presence of a lone pair of electrons in As atoms allows their oxidation, a plausible etching mechanism would involve the oxidation of $\mathrm{As}^{3-}$ to $\mathrm{As}^{3+}$ and $\mathrm{As}^{5+}$ to produce arsenous and arsenic acids, while fluorine ions withdraw the gallium ions, accordingly to the following reactions:

$$
\begin{aligned}
& \mathrm{GaAs}+3 \mathrm{H}_{2} \mathrm{O}_{2}+3 \mathrm{HF} \rightarrow \mathrm{GaF}_{3}+\mathrm{H}_{3} \mathrm{AsO}_{3}+3 \mathrm{H}_{2} \mathrm{O} \\
& \mathrm{GaAs}+4 \mathrm{H}_{2} \mathrm{O}_{2}+3 \mathrm{HF} \rightarrow \mathrm{GaF}_{3}+\mathrm{H}_{3} \mathrm{AsO}_{4}+4 \mathrm{H}_{2} \mathrm{O}
\end{aligned}
$$

The reactivity of $\mathrm{Ga}$ and As is also linked to the number of bonds that they form with the underlying crystal. ${ }^{51}$ When reactive arsenic is etched away from the (111) plane, it reveals a layer of gallium atoms singularly bounded to the crystal, which can be complexed by the fluorine ions. On the other hand, Ga atoms laying on (1 111$)$ and $(1 \overline{3} 1)$ surfaces have respectively two and three bonds with the crystal and are therefore less reactive, slowing down the etch rate (see Supporting Information Fig. S4).

Fig. 2 shows a schematic of the proposed etching mechanism that accounts for the different reactivity of the species involved. Here, a gold nanoparticle laying on the GaAs (111)B surface is oxidized in an acidic environment by $\mathrm{H}_{2} \mathrm{O}_{2}$ to form gold ions (1). ${ }^{52}$ The resulting $\mathrm{Au}^{3+}$ and $\mathrm{Au}^{1+}$ ions diffuse along the surface until they encounter a reactive arsenic site and selectively oxidize it producing $\mathrm{Au}^{0}$ and arsenous and arsenic acids (2). While the newly precipitated gold is subsequently reduced by $\mathrm{H}_{2} \mathrm{O}_{2}$ and becomes available for the oxidation of another reactive site, gallium atoms belonging to the (111)A plane can be complexed by the fluorine ions, thus allowing diffusion of the metal catalyst to the inner surface (3). The process continues until the $<111>$ and $<311>$ monoatomic gallium planes are exposed at the surface $(4,5)$. To confirm this mechanism, a (111)B GaAs surface was etched without catalyst. In this case, no structure attributable to a plane dependent etch rate is discernible on the resulting surface (see Supporting Information Fig. S5). Note that previous works that made use of continuous metal film as etching catalyst $^{33-35,53}$ could not identify the crystal plane dependent etching mechanism since gold diffusion to the active sites is inhibited by the catalyst film itself. ${ }^{32,54}$ 


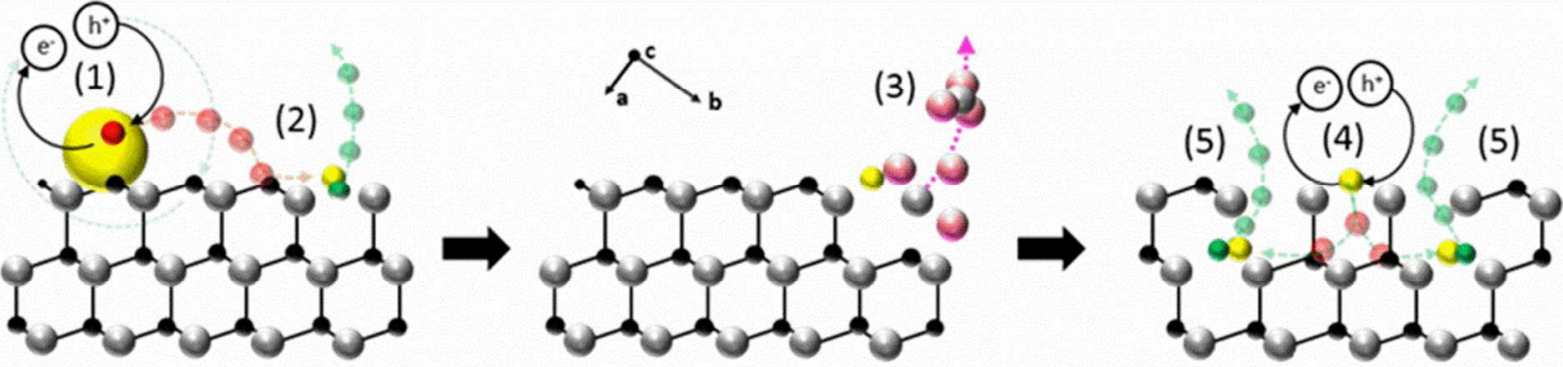

$\rightarrow \mathrm{Au}^{0} \bullet \mathrm{Au}^{3 *} \bullet \mathrm{As}^{5 \cdot} \cdot \mathrm{As}^{3 *} / \mathrm{As}^{5 *} \cup \mathrm{Ga}^{3+} \cup \mathrm{F}$

Figure 2: Schematic of the etching process of a (111)B GaAs plane assisted by a gold nanoparticle. (1) Oxidation of the $\mathrm{Au}$ nanoparticle by $\mathrm{H}_{2} \mathrm{O}_{2}$ to form $\mathrm{Au}$ ions; (2) Diffusion of $\mathrm{Au}^{3+}$ and $\mathrm{Au}^{1+}$ ions and oxidation of a reactive $\mathrm{As}$ site to produce $\mathrm{Au}^{0}$ and arsenous and arsenic acids $\left(\mathrm{H}_{3} \mathrm{AsO}_{3}\right.$ and $\left.\mathrm{H}_{3} \mathrm{AsO}_{4}\right)$; (3) Reduction of precipitated $\mathrm{Au}$ by $\mathrm{H}_{2} \mathrm{O}_{2}$ (which becomes available for the oxidation of another reactive site), and complexation of $\mathrm{Ga}$ atoms in the (111)A plane by $\mathrm{F}^{-}$ions allow diffusion of the metal catalyst to the inner surface; $(4,5)$ Iteration of the process leads to the exposure of the $<111>$ and $<311>$ monoatomic gallium planes.

Etched GaAs samples appear completely black (see insets of Figs. 3c and 3d), a very desirable property for absorber layers in photovoltaic and photodetection applications. This is due to the nearly perfect suppression of reflectance by the roughened surface, very much alike black $S i$ in which silicon nanowires are formed at the surface by MACE catalysed by $\mathrm{AgNO}_{3}$ in $\mathrm{HF}$ and $\mathrm{H}_{2} \mathrm{O}_{2}$ (see Experimental Section and Supporting Information Fig. S6). Fig. 3a compares the antireflective properties of black GaAs to those of a reference black Si sample. Compared to polished Si surfaces with reflectance between 0.62 and 0.90 in the range of 550 to $1150 \mathrm{~nm}$ (grey line in Fig. 3a), the reflectance of black Si oscillates from a minimum of $\sim 0.04$ to a maximum of $\sim 0.2$, corresponding to a $\sim 3$ to 20 -fold reduction. The low surface reflectance of black $S i$ is due to the low refractive index effective medium formed by the nanowire layer, which is also responsible for the pronounced spectral oscillations with typical Fabry-Perot pattern ${ }^{55}$ (orange line in Fig. 3a). In comparison, the reflectance of black GaAs (black and green lines in Fig. 3a) is between $\sim 25-60$ times lower than polished GaAs (red line in Fig. 3a). On average, the antireflective properties of black GaAs are $\sim 2$ to 10 times better than black Si (Supporting Information Fig. S7). Note that similar results were also obtained by diffuse reflectance measurements in an integrating sphere (Supporting Information Fig. S8). As shown in the following, the nearly perfect antireflection properties of black GaAs stem from a completely different optical mechanism than refractive index matching in black Si.

Fig. $3 \mathrm{~b}$ details the specular reflectance properties of the two types of etched GaAs samples. While both samples effectively reduce the reflectance of polished GaAs (red line in Fig. 3a), both above (absorbing 
region) and below the band gap at $890 \mathrm{~nm}(1.4 \mathrm{eV})$, etched GaAs (111)B has much lower reflectance than etched GaAs (100). The lack of an interference pattern in the spectra of etched GaAs samples suggests that suppression of reflectance is due to light scattering from the micro-structured surface rather than to refractive index matching by a surface effective medium like in the case of black Si (See Supporting Information Fig. S6). To further confirm this hypothesis, we plotted the reflectance collected at $800 \mathrm{~nm}$ as a function of the inclination angle of the faceted surface of the three samples (inset of Fig. $3 b)$. The reflectance of the polished surface $\left(0^{\circ}\right)$ is the highest, and decreases exponentially with the inclination angle of the facets on the etched surfaces $\left(26^{\circ}\right.$ for the $(100)$ and $79^{\circ}$ for the (111)B surface, see also Fig. 1). This shows that geometrical light trapping induced by the specific geometry of the surface microfacets of black GaAs is the primary reflection suppression mechanism. Fig. 3c compares the nearinfrared reflectance of polished and etched (111)B GaAs surfaces. While polished GaAs is highly reflective and spectrally flat throughout the entire near-infrared range (red line), the reflectance of etched GaAs varies between 0.08 at $1200 \mathrm{~nm}$ and 0.05 at $2600 \mathrm{~nm}$ (black line), corresponding to a 100 fold decrease compared to the polished surface. This makes black GaAs very interesting also for near-infrared photodetector applications.
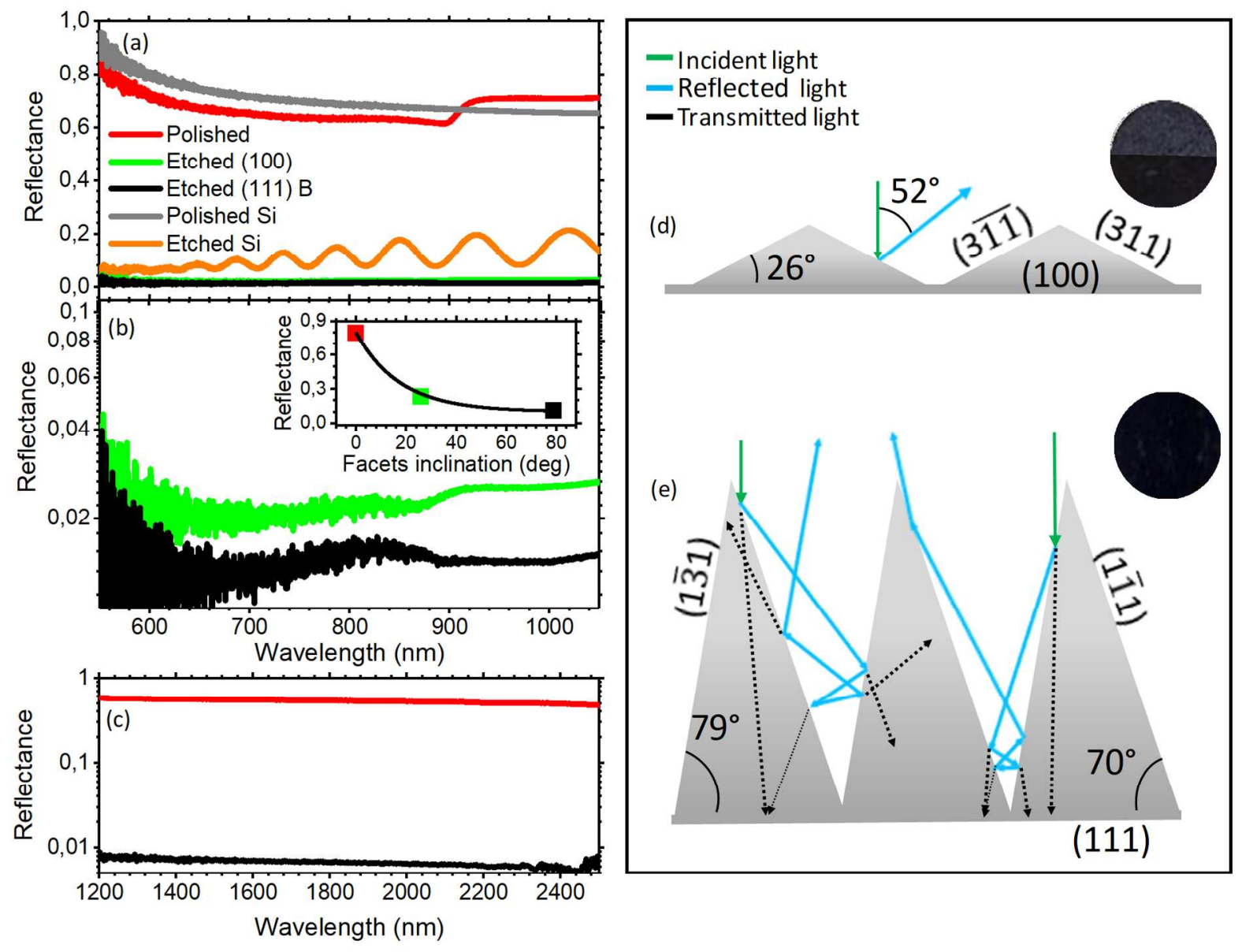
Figure 3: a) Normal incidence reflectance of polished (red line) and etched (100) (green lines) and (111)B (black lines) GaAs surfaces and of polished (grey line) and etched (orange line) Si surfaces. b) Magnified view of the reflectance spectra of the two GaAs etched surfaces. The inset shows the experimental (squares) and fitted (black line) dependence of the GaAs reflectance collected at $800 \mathrm{~nm}$ on the angle of inclination of the faceted surface. c) Normal incidence reflectance of polished (red line) and etched (111)B (black lines) GaAs surfaces in the near infrared spectral region. d, e) Geometrical raytracing analysis of the light path within ideal (100) (c) and (111)B (d) micro-faceted surfaces. The insets of $\mathrm{c}$ and $\mathrm{d}$ show the overlaid photograph of the etched wafers taken at different collection angles.

A simple ray-tracing analysis of the incident light path within the (111)B and (100) etched surfaces provides a qualitative explanation of the light trapping process (Figs. 3d and 3e). Fig. 3d shows an ideal representation of the (1 $\overline{1} 1),(1 \overline{3} 1)$ and (111) facets on the etched (111)B surface (refer to Fig. 1c), while Fig. 3e depicts the (3i1) and (311) facets on the (100) etched surface (refer to Fig. 1f). The microfacets on (100) etched surfaces reflect normal incident light at $\sim 52^{\circ}$, resulting in strong light scattering and minimal light trapping. Conversely, the $(1 \overline{1} 1)$ and $(1 \overline{3} 1)$ microfacets on (111)B etched surfaces redirect specularly reflected normal incidence beams toward the bottom of the valleys between facets, which undergo multiple reflections before leaving the surface. Therefore, the larger inclination of surface microfacets explains the superior antireflective characteristics of etched GaAs (111)B compared to (100).

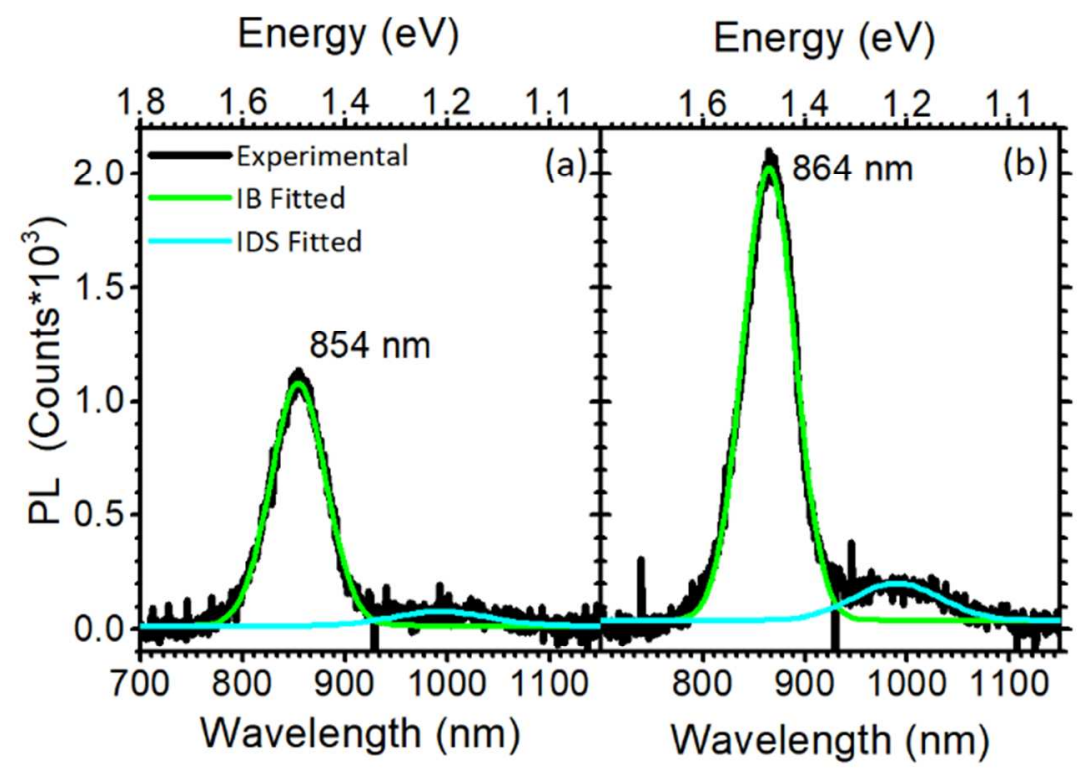

Figure 4: Photoluminescence emission spectra of a) polished and b) etched (111)B GaAs samples (black lines). The green and cyan lines are Gaussian fits to the data corresponding to conduction to valence band (CV) transitions and intra-gap transitions from defect states (IDS), respectively.

Last, photoluminescence spectra and quantum yield measurements were used to gauge the effectiveness of light trapping in black GaAs. Fig. 4 compares the emission spectra of polished GaAs (Fig. 4a) and 
etched GaAs (111)B (Fig. 4b) under $559 \mathrm{~nm}$ excitation. Both samples show two emission peaks in the near infrared range, below the band gap. The most intense peak (at 853 and $864 \mathrm{~nm}$ in polished and etched GaAs, respectively) is due to the conduction to valence band interband transition (CV), ${ }^{56-57}$ while the weaker peak (1010 $\mathrm{nm}$ in both samples) is assigned to transitions from intragap defect states (IDS), commonly generated from silicon n-type doping. ${ }^{56-57}$ The $13 \mathrm{~nm}(\sim 24 \mathrm{meV})$ shift of the CV peak and the enhancement of the IDS emission may be ascribed to the formation of new species (e.g. $\mathrm{Ga}_{2} \mathrm{O}_{2}$ and $\mathrm{As}_{2} \mathrm{O}_{3}$ ) on the etched surface, or to the formation of defects and increase of porosity, which are known to cause strain and lattice distortion. ${ }^{58}$

Table 1: Absorption (Abs) at $559 \mathrm{~nm}$ and total $\left(\eta_{\text {tot }}\right)$, interband transition $\left(\eta_{\mathrm{CV}}\right)$ and intraband surface defect state transition ( $\left.\eta_{\text {IDS }}\right)$ photoluminescence external quantum efficiency of polished and etched (111)B GaAs.

\begin{tabular}{r|cccc} 
& \multicolumn{2}{|c}{ Abs } & & \\
& $\left(\lambda_{\text {ex }}=\mathbf{5 5 9} \mathbf{~ n m}\right)$ & $\eta_{\text {tot }}(\mathbf{\%})$ & $\eta_{\mathbf{C V}}(\mathbf{\%})$ & $\eta_{\text {IDS }}(\mathbf{\%})$ \\
\hline Etched & 0.9 & $3.6 * 10^{-2}$ & $2.6 * 10^{-2}$ & $1.0 * 10^{-2}$ \\
Polished & 0.5 & $3.6 * 10^{-2}$ & $3.2 * 10^{-2}$ & $0.4 * 10^{-2}$ \\
\hline Ratio & 1.8 & 1.0 & 0.8 & 2.5 \\
$($ Etched/Polished $)$ & & & &
\end{tabular}

Notably, the photoluminescence intensity of black GaAs is strongly enhanced compared the polished sample. While the total photoluminescence quantum yield $\left(\eta_{\mathrm{t}}\right)^{59-60}$ remains substantially unchanged after etching, the light absorption (Abs) nearly doubles (Table 1, see Experimental section for measurement details). The increase of light absorption, therefore, largely accounts for the twofold enhancement of integrated emission intensity seen in Figure 4. Interestingly, in the etched sample, the efficiency of the CV transition $\left(\eta_{\mathrm{cv}}\right)$ decreases by a $20 \%$, while the efficiency of the IDS transition ( $\left.\eta_{\text {IDS }}\right)$ is 2.5 times more efficient, yet the total quantum yield remains unaltered. This indicates that, upon absorption, a higher percentage of photoexcitations in black GaAs contribute to radiative intragap surface defect state transitions than to bulk interband recombination.

\section{Conclusions}

In conclusion, we demonstrated a new metal-assisted chemical etching process to create nearly perfect antireflection micro-structured GaAs surfaces in the visible and near-infrared spectral regions (black 
GaAs). This solution-based process relies on a crystal plane dependent etch rate, which slows down at monoatomic gallium $<111>$ and $<311>$ crystallographic orientations regardless of the planes exposed to the etchant. Therefore, etching of the (100) or (111)B surfaces generates highly sloped microfacets which induce efficient light scattering and geometrical light trapping within the semiconductor surface, resulting in up to twofold enhancement of light absorption. We have shown that, on average, the antireflective properties of black GaAs are 2 to 10 times better than black $S i$, in which the reduction of surface reflectivity is induced by a refractive index matched surface nanowire layer, rather than by geometrical light trapping. As a result of increased absorption, the intensity of photoluminescence emitted by black GaAs doubles without affecting the external quantum efficiency, even though interband radiative recombination is reduced in favour of intragap surface defect state transitions. Given its simplicity and scalability, we believe that this method provides a viable way to increase the absorption of III-V semiconductors by microstructuring, and could be readily implemented into the production workflow of solar cells and photodetectors to increase their efficiency.

\section{ASSOCIATED CONTENT}

Supporting Information. Metal Assisted Chemical Etching (MACE) process. Electroldeless deposition of gold nanoparticles. MACE of (100) and (211) GaAs crystalline planes. Slow etching rate planes. Non-catalyzed etching of GaAs, Black Silicon reference; Antireflective properties, Diffuse reflectance measurements of polished and etched GaAs.

\section{AUTHOR INFORMATION}

\section{Corresponding Author}

* E-mail: csoci@ntu.edu.sg

\section{Author Contributions}

The manuscript was written through contributions of all authors.

\section{ACKNOWLEDGMENTS}

Research was supported by the Ministry of Education (MOE2011-T3-1-005, MOE2016-T2-1-052 and MOE2013-T2-1-044) and by the National Research Foundation (NRF-CREATE Singapore-Berkeley Research Initiative for Sustainable Energy, SinBeRISE) of Singapore. We also acknowledge support from the European Commission H2020 programme for the ETN SYNCHRONICS funded under grant agreement 643238. FC is a Royal Society Wolfson Foundation Research Merit Award. 


\section{REFERENCES}

(1) Savin, H.; Repo, P.; von Gastrow, G.; Ortega, P.; Calle, E.; Garín, M.; Alcubilla, R. Black Silicon Solar Cells with Interdigitated Back-Contacts Achieve 22.1\% Efficiency. Nat. Nanotechnol. 2015, $10(7), 624-628$.

(2) Otto, M.; Algasinger, M.; Branz, H.; Gesemann, B.; Gimpel, T.; Füchsel, K.; Käsebier, T.; Kontermann, S.; Koynov, S.; Li, X.; Naumann, V.; Oh, J.; Sprafke, A. N.; Ziegler, J.; Zilk, M.; Wehrspohn, R. B. Black Silicon Photovoltaics. Adv. Opt. Mater. 2015, 3 (2), 147-164.

(3) Tang, Z.; Tress, W.; Inganäs, O. Light Trapping in Thin Film Organic Solar Cells. Mater. Today 2014, 17 (8), 389-396.

(4) Lin, S.-H.; Su, Y.-H.; Cho, H.-W.; Kung, P.-Y.; Liao, W.-P.; Wu, J.-J. Nanophotonic Perovskite Solar Cell Architecture with a Three-Dimensional $\mathrm{TiO}_{2}$ Nanodendrite Scaffold for Light Trapping and Electron Collection. J. Mater. Chem. A 2016, 4 (3), 1119-1125.

(5) Du, Q. G.; Shen, G.; John, S. Light-Trapping in Perovskite Solar Cells. AIP Advances 2016, 6 (6), 065002.

(6) Garnett, E.; Yang, P. Light Trapping in Silicon Nanowire Solar Cells. Nano Lett. 2010, 10 (3), 1082-1087.

(7) Da, Y.; Xuan, Y.; Li, Q. From Light Trapping to Solar Energy Utilization: A Novel PhotovoltaicThermoelectric Hybrid System to Fully Utilize Solar Spectrum. Energy 2016, 95, 200-210.

(8) Brongersma, M. L.; Cui, Y.; Fan, S. Light Management for Photovoltaics Using High-Index Nanostructures. Nat. Mater. 2014, 13 (5), 451-460.

(9) Leung, S.-F.; Zhang, Q.; Xiu, F.; Yu, D.; Ho, J. C.; Li, D.; Fan, Z. Light management with nanostructures for optoelectronic devices. J. Phys. Chem. Lett. 2014, 5 (8), 1479-1495.

(10) Lova, P.; Soci, C. Nanoimprint Lithography: Toward Polymer Photonic Crystals. In Organic and hybrid photonic crystals; Comoretto, D., Ed.; Springer: Cham, 2015; Chapter 187-212, p 493.

(11) Lova, P.; Cortecchia, D.; S. Krishnamoorthy, H. N.; Giusto, P.; Bastianini, C.; Bruno, A.; Comoretto, D.; Soci, C. Engineering the Emission of Broadband 2D Perovskites by Polymer Distributed Bragg Reflectors. ACS Photonics 2018, 5 (3), 867-874.

(12) Miller, O. D.; Yablonovitch, E.; Kurtz, S. R. Strong Internal and External Luminescence As Solar Cells Approach the Shockley-Queisser Limit. IEEE J. Photovolt. 2012, 2 (3), 303-311.

(13) Li, X.; Bohn, P. W. Metal-Assisted Chemical Etching in $\mathrm{HF} / \mathrm{H}_{2} \mathrm{O}_{2}$ Produces Porous Silicon. Appl. Phys. Lett. 2000, 77 (16), 2572-2574.

(14) Chartier, C.; Bastide, S.; Lévy-Clément, C. Metal-Assisted Chemical Etching of Silicon in HF$\mathrm{H}_{2} \mathrm{O}_{2}$. Electochim. Acta 2008, 53 (17), 5509-5516.

(15) Peng, K.; Lu, A.; Zhang, R.; Lee, S.-T. Motility of Metal Nanoparticles in Silicon And Induced Anisotropic Silicon Etching. Adv. Funct. Mater. 2008, 18 (19), 3026-3035.

(16) Zhang, M.-L.; Peng, K.-Q.; Fan, X.; Jie, J.-S.; Zhang, R.-Q.; Lee, S.-T.; Wong, N.-B. Preparation of Large-Area Uniform Silicon Nanowires Arrays Through Metal-Assisted Chemical Etching. $J$. Phys. Chem. B 2008, 112 (12), 4444-4450.

(17) Jansen, H. V.; Boer, M. J. d.; Unnikrishnan, S.; Louwerse, M. C.; Elwenspoek, M. C. Black Silicon Method X: A Review on High Speed and Selective Plasma Etching of Silicon with Profile Control: An In-Depth Comparison Between Bosch and Cryostat Drie Processes as a Roadmap to Next Generation Equipment. J. Micromech. Microeng. 2009, 19 (3), 033001. 
(18) Srivastava, S. K.; Kumar, D.; Singh, P. K.; Kar, M.; Kumar, V.; Husain, M. Excellent Antireflection Properties of Vertical Silicon Nanowire Arrays. Sol. Energy Mater. Sol. Cells 2010, 94 (9), 1506-1511.

(19) Huang, Z.; Geyer, N.; Werner, P.; de Boor, J.; Gosele, U. Metal-Assisted Chemical Etching of Silicon: a Review. Adv. Mater. 2011, 23 (2), 285-308.

(20) Agnieszka, K.; Seán, T. B. Metal-Assisted Chemical Etching Using Sputtered Gold: A Simple Route to Black Silicon. Sci. Technol. Adv. Mater. 2011, 12 (4), 045001.

(21) Smith, Z. R.; Smith, R. L.; Collins, S. D. Mechanism of Nanowire Formation in Metal Assisted Chemical Etching. Electochim. Acta 2013, 92, 139-147.

(22) Rezvani, S. J.; Pinto, N.; Boarino, L. Rapid Formation of Single Crystalline Ge Nanowires by Anodic Metal Assisted Etching. Cryst. Eng. Comm 2016, 18 (40), 7843-7848.

(23) Elwenspoek, M.; Lindberg, U.; Kok, H.; Smith, L. In Wet Chemical Etching Mechanism of Silicon, Micro Electro Mechanical Systems, 1994, MEMS '94, Proceedings, IEEE Workshop on, 1994; 1994; pp 223-228.

(24) Baca, A. G.; Ashby, C. I. H., Fabrication of GaAs Devices. Institution of Engineering and Technology, EMIS processing series Bodmin, UK, 2005.

(25) Unagami, T. Formation mechanism of porous silicon layer by anodization in HF solution. $J$. Electrochem. Soc. 1980, 127 (2), 476-483.

(26) Peng, K.; Xu, Y.; Wu, Y.; Yan, Y.; Lee, S.-T.; Zhu, J. Aligned Single-Crystalline Si Nanowire Arrays for Photovoltaic Applications. Small 2005, 1 (11), 1062-1067.

(27) Liu, K.; Qu, S.; Zhang, X.; Wang, Z. Anisotropic Characteristics and Morphological Control of Silicon Nanowires Fabricated by Metal-Assisted Chemical Etching. J Mater Sci 2013, 48 (4), 1755-1762.

(28) Song, Y.; Choi, K.; Jun, D.-H.; Oh, J. Nanostructured GaAs Solar Cells via Metal-Assisted Chemical Etching of Emitter Layers. Opt. Express 2017, 25 (20), 23862-23872.

(29) Fang, H.; Wu, Y.; Zhu, J. H. Silver Catalysis in the Fabrication of Silicon Nanowire Arrays. Nanotechnology 2006, 17 (3), 3768.

(30) Yae, S.; Kawamoto, Y.; Tanaka, H.; Fukumoro, N.; Matsuda, H. Formation of Porous Silicon by Metal Particle Enhanced Chemical Etching in HF Solution and its Application for Efficient Solar Cells. Electrochem. Commun. 2003, 5 (8), 632.

(31) Asoh, H.; Imai, R.; Hashimoto, H. Au-Capped GaAs Nanopillar Arrays Fabricated by MetalAssisted Chemical Etching. Nanoscale Res. Lett. 2017, 12 (1), 444.

(32) Cheung, H.-Y.; Lin, H.; Xiu, F.; Wang, F.; Yip, S.; Ho, J. C.; Wong, C.-Y. Mechanistic Characteristics of Metal-Assisted Chemical Etching in GaAs. J. Phys. Chem. C 2014, 118 (13), 6903-6908.

(33) Yasukawa, Y.; Asoh, H.; Ono, S. Morphological Control if Periodic GaAs Hole Arrays by Simple Au-Mediated Wet Etching. J. Electrochem. Soc. 2012, 159 (5), D328-D332.

(34) DeJarld, M.; Shin, J. C.; Chern, W.; Chanda, D.; Balasundaram, K.; Rogers, J. A.; Li, X. Formation of High Aspect Ratio GaAs Nanostructures with Metal-Assisted Chemical Etching. Nano Lett. 2011, 11 (12), 5259-5263.

(35) Mohseni, P. K.; Kim, S. H.; Zhao, X.; Balasundaram, K.; Kim, J. D.; Pan, L.; Rogers, J. A.; Coleman, J. J.; Li, X. Gaas Pillar Array-Based Light Emitting Diodes Fabricated by MetalAssisted Chemical Etching. J. App. Phys. 2013, 114 (6), 064909-6. 
(36) Asoh, H.; Suzuki, Y.; Ono, S. Metal-Assisted Chemical Etching of GaAs Using Au Catalyst Deposited on The Backside Of A Substrate. Electochim. Acta 2015, 183, 8-14.

(37) Lee, A. R.; Kim, J.; Choi, S.-H.; Shin, J. C. Formation of Three-Dimensional GaAs Microstructures by Combination of Wet and Metal-Assisted Chemical Etching. Phys. Status Solidi RRL 2014, 8 (4), 345-348.

(38) Ono, S.; Kotaka, S.; Asoh, H. Fabrication and Structure Modulation of High-Aspect-Ratio Porous Gaas Through Anisotropic Chemical Etching, Anodic Etching, and Anodic Oxidation. Electochim. Acta 2013, 110, 393-401.

(39) Song, Y.; Oh, J. Fabrication of Three-Dimensional GaAs Antireflective Structures by MetalAssisted Chemical Etching. Sol. Energy Mater. Sol. Cells 2016, 144, 159-164.

(40) Cowley, A.; Steele, J. A.; Byrne, D.; Vijayaraghavan, R. K.; McNally, P. J. Fabrication and Characterisation of GaAs Nanopillars Using Nanosphere Lithography and Metal Assisted Chemical Etching. RSC Adv. 2016, 6 (36), 30468-30473.

(41) Kong, L.; Song, Y.; Kim, J. D.; Yu, L.; Wasserman, D.; Chim, W. K.; Chiam, S. Y.; Li, X. Damage-Free Smooth-Sidewall InGaAs Nanopillar Array by Metal-Assisted Chemical Etching. ACS Nano 2017, 11 (10), 10193-10205.

(42) Glinsner, T.; Kreindl, G. Lithography, InTech: 2010; p 656.

(43) Peng, K.; Zhang, M.; Lu, A.; Wong, N.-B.; Zhang, R.; Lee, S.-T. Ordered Silicon Nanowire Arrays via Nanosphere Lithography and Metal-Induced Etching. App. Phys. Lett. 2007, 90 (16), 163123-3.

(44) Radiative Transitions Induced In Gallium Arsenide by Modest Heat Treatment. J. Appl. Phys. 1980, 51 (1), 619-624.

(45) Im, K.; Kang, J.-H.; Park, Q. H. Universal Impedance Matching and The Perfect Transmission of White Light. Nat. Photonics 2018, 12 (3), 143-149.

(46) Momma, K.; Izumi, F. VESTA 3 for Three-Dimensional Visualization of Crystal, Volumetric And Morphology Data. J. Appl. Crystallogr. 2011, 44 (6), 1272-1276.

(47) http://www.crystallography.net/result.php retrieved on 21 May 2018.

(48) de Mello, J. C.; Wittmann, H. F.; Friend, R. H. An Improved Experimental Determination of External Photoluminescence Quantum Efficiency. Adv. Mater. 1997, 9 (3), 230-232.

(49) Manfredi, G.; Lova, P.; Di Stasio, F.; Krahne, R.; Comoretto, D. Directional Fluorescence Spectral Narrowing in All-Polymer Microcavities Doped with CdSe/CdS Dot-in-Rod Nanocrystals. ACS Photonics 2017, 4 (7), 1761-1769.

(50) Lova, P.; Grande, V.; Manfredi, G.; Patrin, M.; Herbst, S.; Würthner, F.; Comoretto, D. AllPolymer Photonic Microcavities Doped with Perylene Bisimide J-Aggregates. Adv. Opt. Mater. 2017, 5 (21), 1700523.

(51) MacFadyen, D. N. On the Preferential Etching of GaAs by $\mathrm{H}_{2} \mathrm{SO}_{4} \square \mathrm{H}_{2} \mathrm{O}_{2} \square \mathrm{H}_{2} \mathrm{O} J$. Electrochem. Soc. 1983, 130 (9), 1934-1941.

(52) Mikhael, B.; Elise, B.; Xavier, M.; Sebastian, S.; Johann, M.; Laetitia, P. New Silicon Architectures by Gold-Assisted Chemical Etching. ACS Appl. Mater. Interfaces 2011, 3 (10), 3866-3873.

(53) Bienaime, A.; Elie-Caille, C.; Leblois, T. Micro Structuration of GaAs Surface by Wet Etching: Towards a Specific Surface Behavior. J. Nanosci. Nanotechnol. 2012, 12 (8), 6855-6863. 
(54) Song, Y.; Oh, J. Thermally Driven Metal-Assisted Chemical Etching of GaAs with In-Position and Out-Of-Position Catalyst. J. Mater. Chem. A 2014, 2 (48), 20481-20485.

(55) Sailor, M. J. Porous Silicon in Practice: Preparation, Characterization and Applications, John Wiley \& Sons: 2012.

(56) Ky, N. H.; Reinhart, F. K. Amphoteric Native Defect Reactions in Si-Doped GaAs. J. Appl. Phys. 1998, 83 (2), 718-724.

(57) Ha, Y.-K.; Lee, C.; Kim, J.-E.; Park, H. Y.; Kim, S.; Lim, H.; Kim, B.-C.; Lee, H.-C. Defect Luminescence in Heavily Si-Doped n-and p-Type GaAs. J. Korean Phys. Soc. 2000, 36 (1), $42-$ 48.

(58) Sabataityt, J.; Šimkien, I.; Bendorius, R. A.; Grigoras, K.; Jasutis, V.; Pačebutas, V.; Tvardauskas, H.; Naudžius, K. Morphology and Strongly Enhanced Photoluminescence of Porous GaAs Layers Made by Anodic Etching. Mater. Sci. Eng., Proc. Conf. 2002, 19 (1-2), 155-159.

(59) Takao, N.; Kyo-ichiro, F.; Taiji, O. Improvement of Quantum Efficiency in Gallium Arsenide Electroluminescent Diodes. Jpn. J. Appl. Phys. 1967, 6 (6), 665.

(60) Nelson, R. J.; Sobers, R. G. Minority $\square$ Carrier Lifetimes and Internal Quantum Efficiency of Surface $\square$ Free GaAs. J. Appl. Phys. 1978, 49 (12), 6103-6108. 
For Table of Contents only

Table of Contents artwork

2

3 4

5

6

7

8

9

10

11

12

13

14

15

16

17

18

19

20

21

22

23

24

25

26

27

28

29

30

31

32

33

34

35

36

37

38

39

40

41

42

43

44

45

46

47

48

49

50

51

52

53

54

55

56

57

58

59

60

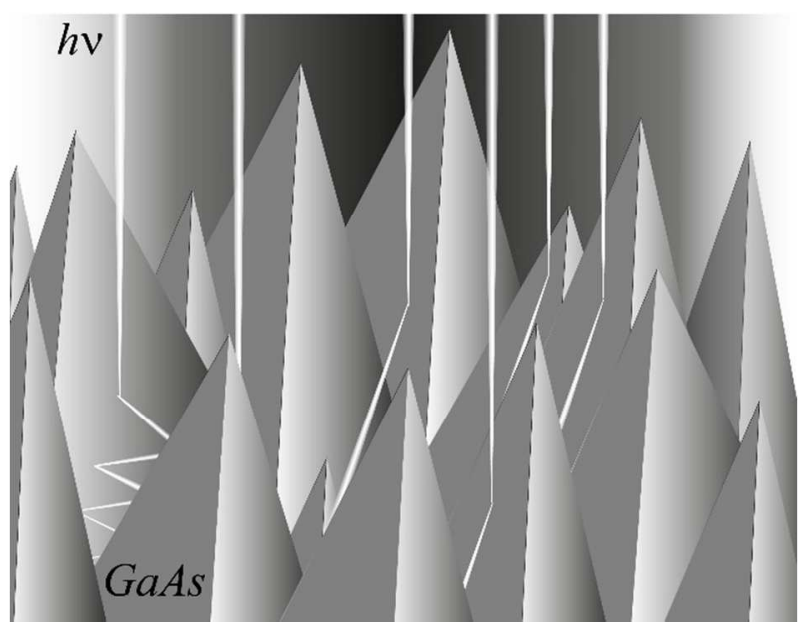

ACS Paragon Plus Environment 